\title{
SUMMARY OF THE THIRD INTERNATIONAL WORKSHOP ON IRON AND COPPER HOMEOSTASIS
}

$\mathrm{T}$ This special issue of Biological Research contains presentations from the Third International Workshop on Iron and Copper Homeostasis (TIWICH), held in Viña de Mar, Chile 9-13 December, 2004. TIWICH, which received the generous funding from the National Institute of Health of the United States of America and the Millennium Institute for Advanced Studies in Cell Biology and Biotechnology of Chile, followed two other successful meetings, the First (1999) and Second (2001) International Workshops on Iron and Copper Homeostasis .

\section{Why hold an iron-copper conference}

Living organisms have a love-hate relationship with iron and copper. Both are nearly universally required nutrients whose excess or deficiency cause impaired cellular functions and eventually cell death. This parallelism in itself would justify holding a workshop on the two metals, but they also have extensive metabolic interactions.

Better understanding of the interactions between iron and copper homeostasis needs to be developed, since their metabolisms are intimately intertwined. In humans, iron or copper deficiency results in diminished work capacity, reduced intellectual capacity, diminished body growth, alterations in bone mineralization, diminished immune response, etc. Although it has been known for many years that systemic copper deficiency generates cellular iron deficiency, the molecular basis for the iron-copper relationships are just now becoming known. Copper is a prosthetic group of the ferroxidase involved in high affinity iron transport in yeast. Similarly in mammals, deficiency in ceruloplasmin, a plasma copper ferroxidase, induces systemic iron deficiency with iron retention in some tissues. Hephaestin apparently plays an almost overlapping role at the serosal side of the enterocyte. The divalent metal transporter DMT1 transports both iron and copper. These intertwinings are probably a basis for extensive regulatory interactions that make the list one that continued to grow at TIWICH.

We already know that excess iron or copper produces oxidative damage and cell death. Presumably this damage occurs because labile iron or copper catalyze the production of hydrogen peroxide and the hydroxyl free radical through the Fenton and Haber-Weiss reactions. Preferential targets of the hydroxyl free radical are membrane lipids and the chromatin. The generation of free radicals underlies the free radical mediated theory of aging. Cells with low duplication rate, as neurons, accumulate free radical produced damage until death. Many of the papers from the Workshop expand on this theme.

\section{TIWICH}

In addressing current issues of metal essentiality/toxicity, TIWICH was organized into five main sessions: Homeostasis of Transition Metals; Metal Physiology; Iron and Copper in Neurodegeneration; Physiopathology of Transition Metals; and Genetic and Proteomic Approaches to the Study of Metal Metabolism. The main sessions were highlighted by Keynote Conferences which included issues such as History of IronCopper Interactions; New High Throughput Assays for Assessing Transition Metals in Cells and Biological Fluids; DMT1 as a Multi-metal Transporter; Iron at the Center of Metal/Oxygen Homeostasis; RedoxActive Iron in Alzheimer Brain; Manganese Toxicity; and Similarities between Prokaryote and Eukaryote Copper 
Metabolism. The Workshop also included four poster sessions, in which students had ample occasions to talk with the participating scientists.

We are certain that this issue will be an important addition to the knowledge of the iron/copper field. We also expect that it will be a source of inspiration to scientists and students to carry out further experimentations in a field that is becoming of extreme relevance for the scientific understanding of concepts literally as crucial as life and death.

\section{MARCO T NÚÑEZ}

Laboratory of Iron and Biology of Aging, Department of Biology, Faculty of Sciences, University of Chile, Casilla 653, Santiago, Chile

\section{MICHAEL D GARRICK}

Department of Biochemistry, SUNY at Buffalo, Buffalo, NY 14214 